04,11

\title{
Статистическая теория орбитальных упорядочений в перовскитах на основе трехминимумной двухподрешеточной модели
}

\author{
(C) М.П. Ивлиев, В.П. Сахненко \\ Научно-исследовательский институт физики Южного федерального университета, \\ Ростов-на-Дону, Россия \\ E-mail: ivlievmp@rambler.ru
}

(Поступила в Редакцию 25 мая 2016 г.)

Установленное для ряда перовскитов $\left(\mathrm{LaMnO}_{3}, \mathrm{KCrF}_{3}\right.$ и др.), содержащих ян-теллеровские катионы, существование в высокотемпературной кубической фазе тетрагонально искаженных октаэдров и проведенные нами оценки позволяют рассмотреть наблюдаемые орбитальные упорядочения как фазовые переходы порядок-беспорядок. На основе трехминимумной двухподрешеточной модели определены условия возникновения ферро- и антиферродисторсионных фазовых состояний, описываемых параметрами порядка, относящимися к векторам $\mathbf{k}=0$ и $\mathbf{k}=(1 / 2,1 / 2,1 / 2)$ обратной решетки. Показано, что модель применима для описания фазовых переходов в некоторых ионно-молекулярных кристаллах.

Работа выполнена при поддержке гранта ЮФУ № 213.01-2014/011-ВГ.

\section{1. Введение}

Кристаллы, содержащие катионы с орбитально вырожденным основным состоянием, обладают рядом уникальных физических свойств [1-3]. Важную роль в формировании таких свойств играет возникновение коллективного орбитального упорядочения в подсистеме анионных полиэдров (октаэдров, тетраэдров), искаженных вследствие эффекта Яна-Теллера. Тип орбитального упорядочения во многом определяет структурный мотив, магнитную структуру, упругие свойства [4-6], электропроводность [7].

B перовскитах $\mathrm{RbCuF}_{3}, \mathrm{KCuF}_{3}, \mathrm{KCrF}_{3}, \mathrm{MnF}_{3}$, $\mathrm{LaMnO}_{3}, \mathrm{BiMnO}_{3}$ катионы $\mathrm{Cu}^{2+}, \mathrm{Cr}^{2+}, \mathrm{Mn}^{3+}$ расположены в центре анионных октаэдров. Их электронные конфигурации $-d^{4}\left(\mathrm{Cr}^{2+}, \mathrm{Mn}^{3+}-t_{2 g}^{3} e_{g}^{1}\right)$ и $d^{9}\left(\mathrm{Cu}^{2+}-\right.$ $\left.t_{2 g}^{6} e_{g}^{3}\right)$, при этом уровень $e_{g}$ вырожден. Снятие вырождения осуществляется посредством деформации октаэдра (эффект Яна-Теллера). При растяжении октаэдра вдоль одной из осей $C_{4}$, например вдоль оси $z$, энергия орбитали $d_{z^{2}}\left(d_{2 z^{2}-x^{2}-y^{2}}\right)$ уменьшается, а энергия орбитали $d_{x^{2}-y^{2}}$ увеличивается. Это приводит к стабилизации орбитали $d_{z^{2}}$ (при сжатии будет стабилизироваться орбиталь $\left.d_{x^{2}-y^{2}}\right)$. Для упомянутых ян-теллеровских (ЯТ) катионов деформации оказываются довольно значительными [8]. В перовскитах с такими „сильно деформирующими“ ЯТ-катионами в большинстве случаев возникают упорядочения антиферродисторсионного типа. При этом основной структурный мотив формируется именно орбитальным упорядочением, несмотря на наличие или появление в ряде случаев упорядочений другого типа: ротационных, магнитных и т.п.

Так, в кристаллах $\mathrm{RbCuF}_{3}, \mathrm{KCrF}_{3}$ наблюдается фазовый переход $(Ф П)$ из кубической (пространственная группа $\left.O_{h}^{1}(P m 3 m), Z=1\right)$ в тетрагональную антиферродисторсионно упорядоченную фазу (пр.гр. $D_{4 h}^{18}$ $(I 4 / \mathrm{mcm}), Z=4) \quad[9,10]$. В кристалле $\mathrm{KCuF}_{3}$ сосуще- ствуют две структурные модификации. Это антиферродисторсионно упорядоченные тетрагональные фазы с симметрией $D_{4 h}^{18}$ и (реже) $D_{4 h}^{5}(P 4 / m b m, Z=2)[11]$.

В кристалле $\mathrm{MnF}_{3}$ орбитальное упорядочение типа $D_{4 h}^{18}$ возникает из кубической фазы (пр.гр. $O_{h}^{1}$ ) совместно с ротационным (смятие, tilt), при этом формируется моноклинная фаза (пр.гр. $C_{2 h}^{6}(C 2 / c)$, $Z=4)[12,13]$. В перовскитах $\mathrm{LaMnO}_{3}$ и $\mathrm{BiMnO}_{3}$ параметры порядка, характеризующие орбитальные упорядочения типа $D_{4 h}^{5}$ и $D_{4 h}^{18}$, возникают как „несобственные“ в ротационно искаженных фазах (пр.гр. $D_{2 h}^{16}$ (Pmna), $Z=4$ для $\mathrm{LaMnO}_{3}[14]$ и $C_{2 h}^{6}(C 2 / c), Z=4$ для $\left.\mathrm{BiMnO}_{3}[15]\right)$. При изосимметричном ФП основной структурный мотив этих соединений переформатируется. После перехода он формируется орбитальным упорядочением, а не ротационным.

Экспериментальные исследования перовскитов свидетельствуют о том, что устойчивые искажения октаэдров с ЯТ-катионами существуют при значительно более высоких температурах, чем температуры возникновения дальнего порядка [16-18]. Это позволяет рассмотреть соответствующие ФП как упорядочение в системе тетрагонально искаженных октаэдров, которые в симметричной фазе имеют три равноправные ориентации вдоль направлений [001] кубической решетки. При этом упорядочение трактуется как перераспределение вероятности ориентаций по трем кристаллографически эквивалентным позициям (КЭП) [5,19].

Орбитально упорядоченные фазы с симметрией $D_{4 h}^{1}$ $(P 4 / m m m, \quad Z=1), \quad D_{4 h}^{18} \quad(I 4 / m c m, \quad Z=4) \quad$ и $\quad D_{4 h}^{5}$ $(P 4 / m b m, \quad Z=2)$ индуцированы параметрами порядка (ПП), преобразующимися по неприводимым представлениям $\Gamma_{3}^{+}(\mathbf{k}=0), R_{3}^{+}\left(\mathbf{k}=1 / 2\left(\mathbf{b}_{1}+\mathbf{b}_{2}+\mathbf{b}_{3}\right)\right)$ и $M_{2}^{+}\left(\mathbf{k}=1 / 2\left(\mathbf{b}_{1}+\mathbf{b}_{2}\right)\right) \quad\left(\mathbf{b}_{i}-\right.$ базисные векторы обратной решетки) пространственной группы $O_{h}^{1}(P m 3 m$, $Z=1)$ [20] соответственно. Наиболее распространенными являются упорядочения ферродисторсионного типа 
$(\mathbf{k}=0)$ и упорядочения антиферродисторсионного типа c $\mathbf{k}=1 / 2\left(\mathbf{b}_{1}+\mathbf{b}_{2}+\mathbf{b}_{3}\right)$. Всю совокупность упорядочений $\mathbf{c} \mathbf{k}=0$ и $\mathbf{k}=(1 / 2,1 / 2,1 / 2)$ можно рассмотреть в рамках единого подхода на основе трехминимумной двухподрешеточной модели. В настоящей работе ограничимся статистико-термодинамическим исследованием этой модели. Условия совместного появления фаз с симметрией $D_{4 h}^{5}$ и $D_{4 h}^{18}$ анализировались на основе трехминимумной восьмиподрешеточной модели в [21].

Обычно статистико-термодинамические свойства многоминимумных моделей исследуются на основе приближения среднего поля. В частности, такой подход использовался при исследовании на базе трехминимумной модели ФП в шпинелях [4,22], перовскитах [19,23] и других соединениях [24]. В [25] исследовались некоторые ФП описываемые трехминимумной двухподрешеточной моделью Поттса. В большинстве случаев основное внимание уделялось анализу ФП в конкретных соединениях и подбору параметров модели, позволяющих получить наблюдаемые температуры ФП.

Трехминимумная двухподрешеточная модель „реализуется“ во многих соединениях, существенно различающихся и по составу, и по типу упорядочивающихся частиц. Поэтому для описания на ее основе столь разнородных систем необходимо знать статистикотермодинамические свойства модели в широких интервалах термодинамических и модельных параметров, что и является основной целью настоящей работы.

\section{2. Описание модели}

В качестве модельной системы рассматривается кристалл, имеющий простую кубическую решетку с симметрией $O_{h}^{1}$, в узлах которой расположены ЯТ-катионы. Позиции узлов фиксированы. В симметричной фазе орбитали типа $d_{z^{2}}$ ЯТ-катиона равновероятно ориентированы вдоль одного из трех направлений типа [001].

Введем функции $n_{i}(i=1,2,3)$, характеризующие вероятности ориентации орбитали катиона вдоль направления $i$ (другими словами, вероятности заполнения $i$-й КЭП). Фазовый переход связывается с изменением соотношения между этими вероятностями при изменении термодинамических параметров. Ограничиваясь исследованием ориентационных упорядочений ферро- и антиферродисторсионного типа, относящихся к векторам $\mathbf{k}=0$ и $\mathbf{k}=1 / 2\left(\mathbf{b}_{1}+\mathbf{b}_{2}+\mathbf{b}_{3}\right)$, кристалл можно представить в виде двух одинаковых, взаимопроникающих, по-разному упорядоченных подрешеток, узлы которых чередуются по трем направлениям типа [001].

Функции $n_{i}$ можно представить в виде

$$
\begin{gathered}
n_{1(\mathrm{I}, \mathrm{II})}=\frac{1}{3}\left(1+\frac{2 \eta_{1}}{\sqrt{6}} \pm \frac{2 \tau_{1}}{\sqrt{6}}\right), \\
n_{2(\mathrm{I}, \mathrm{II})}=\frac{1}{3}\left(1-\frac{\eta_{1}}{\sqrt{6}}+\frac{\eta_{2}}{\sqrt{2}} \pm\left(-\frac{\tau_{1}}{\sqrt{6}}+\frac{\tau_{2}}{\sqrt{2}}\right)\right), \\
n_{3(\mathrm{I}, \mathrm{II})}=\frac{1}{3}\left(1-\frac{\eta_{1}}{\sqrt{6}}+\frac{\eta_{2}}{\sqrt{2}} \pm\left(-\frac{\tau_{1}}{\sqrt{6}}-\frac{\tau_{2}}{\sqrt{2}}\right)\right),
\end{gathered}
$$

где I, II - номер подрешетки, $i=1,2,3$ - номер КЭП.
В столбце \pm знак „Плюс “ соответствует первой подрешетке, а знак „минус“ - второй. Функции $\eta$ и $\tau$ выполняют роль параметров порядка, $\eta$ преобразуется по представлению $\Gamma_{3}^{+}(\mathbf{k}=0)$, а $\tau-$ по $R_{3}^{+}$ $(\mathbf{k}=(1 / 2,1 / 2,1 / 2))$. По трансформационным свойствам ПП $\eta$ совпадает с двухкомпонентным тензором однородных одноосных деформаций: $\eta_{1} \sim 2 u_{z z}-u_{y y}-u_{x x}$, $\eta_{2} \sim u_{y y}-u_{x x}$. Из (1) видно, что ПП $\tau$ характеризует разницу, а ПП $\eta$ - однородную составляющую в упорядочении подрешеток.

В этом случае неравновесный термодинамический потенциал (ТП) в расчете на одну частицу каждой из подрешеток трехминимумной двухподрешеточной модели в приближении Горского-Брэгга-Вильямса [26,27] имеет вид

$$
F=\frac{A}{3} \sum_{i=1,2} \tau_{i}^{2}+\frac{B}{3} \sum_{j=1,2} \eta_{j}^{2}+T \sum_{L=\mathrm{I}, \mathrm{II}} \sum_{i=1}^{3} n_{i, L} \ln n_{i, L}
$$

Константы $A$ и $B$ при таком рассмотрении характеризуют взаимодействие между частицами (как прямое, так и косвенное) через подсистему анионов, позиции которых не фиксированы. Эти константы выступают в качестве феноменологических параметров теории.

\section{3. Фазовые состояния трехминимумной двухподрешеточной модели}

Равновесные значения ПП $\eta$ и $\tau$ можно найти, решив систему уравнений состояния

$$
\begin{aligned}
& \frac{\partial F}{\partial \eta_{i}}=0 \quad(i=1,2), \\
& \frac{\partial F}{\partial \tau_{j}}=0 \quad(j=1,2)
\end{aligned}
$$

и выбрав то решение, которому соответствует абсолютный минимум $F$ при заданных значениях $A, B$ и $T$.

Из соображений симметрии система уравнений состояния может иметь помимо симметричного $|\eta|=|\tau|=0$ (пр. гр. $\left.O_{h}^{1}(P m 3 m), Z=1\right)$ два набора решений. Первый возникает вследствие конденсации ПП $\eta$ и характеризуется вектором обратной решетки $\mathbf{k}=0$, второй ПП $\tau \mathbf{c} \mathbf{k}=(1 / 2,1 / 2,1 / 2)$. В первый набор входят два решения типа $\eta_{1}, \eta_{2}=0$ (пр.гр. $\left.D_{4 h}^{1}(P 4 / \mathrm{mmm}), Z=1\right)$ и $\eta_{1}, \eta_{2}$ (пр. гр. $\left.D_{2 h}^{1}(P m m m), Z=1\right)$, описывающие сегнетоэластические фазы с упорядочением ферродисторсионного типа. Во второй набор входят три решения типа $\eta_{1}, \eta_{2}=0, \tau_{1}=0, \tau_{2}$ (пр. гр. $\left.D_{4 h}^{18}(I 4 / \mathrm{mcm}), Z=4\right)$, $\eta_{1}, \eta_{2}=0, \tau_{1}, \tau_{2}=0$ (пр.гр. $\left.D_{4 h}^{17}(I 4 / \mathrm{mmm}), Z=4\right)$ и $\eta_{1}, \eta_{2}, \tau_{1}, \tau_{2}$ (пр. гр. $\left.D_{4 h}^{23}(F m m m), Z=4\right)$, описывающие несобственно сегнетоэластические фазы с упорядочением антиферродисторсионного типа. 


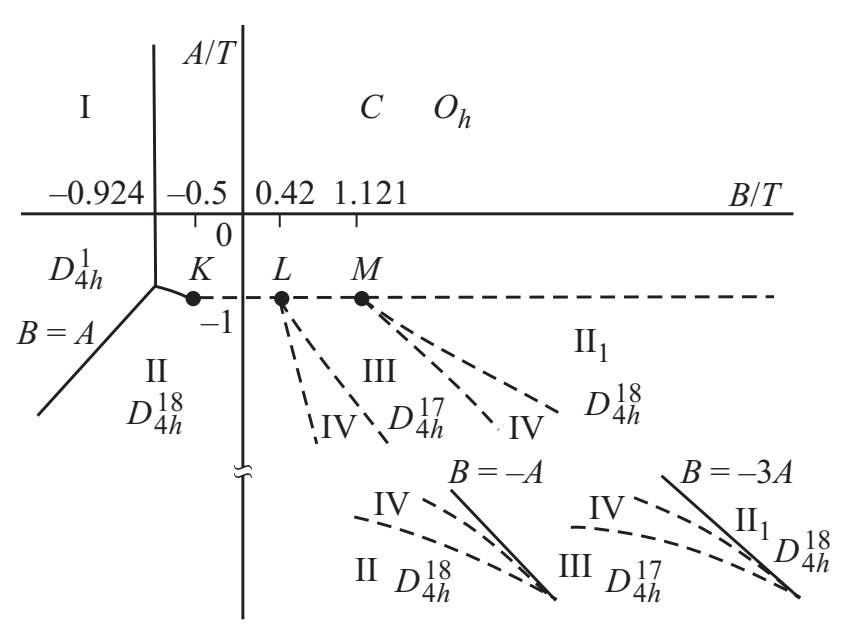

Рис. 1. Диаграмма фазовых состояний, описываемая ТП (2). Сплошными линиями обозначены линии фазовых переходов первого рода, штриховыми - второго рода. Линии $B=-A$ и $B=-3 A-$ асимптоты, к которым сходятся межфазные границы при $T \rightarrow 0$.

Диаграмма фазовых состояний (ФС), описываемая ТП (2), изображена на рис. 1. Симметричная фаза (фаза $C$ ) устойчива в области $A / T \geq-1$, $B / T \geq-1$. Фаза I $-\eta_{1}>0, \eta_{2}=|\tau|=0 \quad\left(n_{1, \mathrm{I}}=n_{1, \mathrm{II}}\right.$, $\left.n_{2, \mathrm{I}}=n_{2, \mathrm{II}}=n_{3, \mathrm{I}}=n_{3, \mathrm{II}}\right) \quad$ - является ферродисторсионно упорядоченной (сегнетоэластической) фазой (пр. гр. $\left.D_{4 h}^{1}\right)$. Она граничит с симметричной по линии ФП первого рода. На этой границе ПП $\eta_{1}$ испытывает „скачок“ от нуля до $\sqrt{6} / 2$. Род перехода и знак ПП однозначно определяются наличием в разложении ТП (2) в ряд по степеням ПП $\eta(|\tau|=0)$ кубического инварианта $\eta_{1}^{3}-3 \eta_{1} \eta_{2}^{2}$ с отрицательным численным коэффициентом.

При $|\tau|=0$ система уравнений $(3),(4)$ может иметь только два решения: устойчивое $\left(\eta_{1}>0\right)$ и неустойчивое $\left(\eta_{1}<0\right)$. Решение $\left(\eta_{1}, 0,0, \tau_{2}\right)$ ответвляется от неустойчивого решения $\left(\eta_{1}<0,0,0,0\right)$, поэтому появившаяся фаза II $\left(n_{1, \mathrm{I}}=n_{1, \mathrm{II}}, n_{2, \mathrm{I}}=n_{3, \mathrm{II}}, n_{3, \mathrm{I}}=n_{2, \mathrm{II}}\right)$, описываемая решением $\left(\eta_{1}<0,0,0, \tau_{2}\right)$, также неустойчива, ее ТП больше ТП фазы І. Эти фазы различаются взаимной ориентацией подрешеток. В фазе I частицы обеих подрешеток ориентированы параллельно, а в фазе II - opтогонально друг другу. Термодинамические потенциалы фаз I и II сравниваются на линии $B=A, A<0$.

В области $A<0, A<B$ критическим является ПП $\tau$. Конденсация $\tau$ приведет к мультипликации ячейки и появлению двух по-разному упорядоченных подрешеток. В этой области термодинамический потенциал фазы II будет меньше, чем термодинамический потенциал фазы I.

Вблизи границы с симметричной фазой, в области, где фазовый перход по ПП $\tau$ является переходом второго рода, ТП (2) можно разложить в ряд по $\eta$ и $\tau$ [28]. Поскольку $\Gamma_{3}^{+} \in\left(R_{3}^{+}\right)^{2}$, ПП $\eta$ будет появляться как вторичный при появлении ПП $\tau$. Из уравнения (3) получаем

$$
f_{1}=\frac{1}{6 b}\left(3 t_{1}^{2}-t_{2}^{2}\right)+\ldots, \quad f_{2}=-\frac{1}{b} t_{1} t_{2}+\ldots,
$$

где

$$
\begin{gathered}
b=\frac{B}{T}+1, \quad f_{1}=\frac{\eta_{1}}{\sqrt{6}}, \quad f_{2}=\frac{\eta_{2}}{\sqrt{2}}, \\
t_{1}=\frac{\tau_{1}}{\sqrt{6}}, \quad t_{2}=\frac{\tau_{2}}{\sqrt{2}} .
\end{gathered}
$$

Подставив в ТП (2), который представлен в виде ряда по степеням ПП $\eta$ и $\tau$, параметры $f$ и $t$ из (5а), а вместо $f$ выражения из (5), переходим к эффективному ТП, зависящему только от $t$ :

$$
\begin{aligned}
\frac{3 F_{\mathrm{eff}}}{T}= & 2 \alpha_{1}\left(3 t_{1}^{2}+t_{2}^{2}\right)+\alpha_{2}\left(3 t_{1}^{2}+t_{2}^{2}\right)^{2} \\
& +\beta_{1}\left(t_{1}^{3}-t_{1} t_{2}^{2}\right)^{2}+\alpha_{3}\left(3 t_{1}^{2}+t_{2}^{2}\right)^{3}+\ldots \\
& +\delta_{12}\left(3 t_{1}^{2}+t_{2}^{2}\right)\left(t_{1}^{3}-t_{1} t_{2}^{2}\right)^{2}+\beta_{2}\left(t_{1}^{3}-t_{1} t_{2}^{2}\right)^{4}+\ldots
\end{aligned}
$$

где

$$
\begin{gathered}
\alpha_{1}=\frac{A}{T}+1, \alpha_{2}=\frac{1}{3}\left(1-\frac{1}{2 b}\right), \beta_{1}=\frac{4}{5}-\frac{3}{b}+\frac{3}{b^{2}}-\frac{1}{2 b^{3}}, \\
\alpha_{3}=\frac{2}{15}-\frac{1}{6 b}+\frac{1}{18 b^{2}}, \delta_{12}=\frac{8}{7}-\frac{6}{b}+\frac{10}{b^{2}}-\frac{6}{b^{3}}+\frac{1}{b^{4}}, \\
\beta_{2}=\frac{8}{11}-\frac{39}{5 b}+\frac{33}{b^{2}}-\frac{285}{4 b^{3}}+\frac{339}{4 b^{4}}-\frac{225}{4 b^{5}} \\
+\frac{104}{5 b^{6}}-\frac{9}{19 b^{7}}+\frac{9}{16 b^{8}}-\frac{1}{32 b^{9}} .
\end{gathered}
$$

Действительно, в области $\alpha_{2} \geq 0(b \geq 0.5, B / T \geq-0.5)$ на линии $\alpha_{1}=0\left(A / T_{c}=-1\right)$ имеет место ФП второго рода, обусловленный конденсацией ПП $\tau . T_{c}=-A-$ температура потери устойчивости симметричной фазы относительно появления ПП $\tau$, аналог температуры Кюри-Вейсса в сегнетоэлектриках. В области $\alpha_{2} \leq 0$ $(b \leq 0.5, B / T \leq-0.5)$ вблизи линии $\alpha_{1}=0$ имеет место ФП первого рода из симметричной фазы $C$ в фазу II. При $b=0.5$ коэффициенты $\alpha_{3}$ и $\beta_{1}-$ положительны, поэтому точка $K$ с координатами $B / T=-0.5, A / T=-1-$ трикритическая точка, в которой линия ФП первого рода из фазы $C$ превращается в линию ФП второго рода. Экстремумы (6) по $\tau$ типа $\left(\tau_{1}, 0\right)$ и $\left(0, \tau_{2}\right)$ описывают два типа тетрагональных фаз с симметрией $D_{4 h}^{17}(I 4 / \mathrm{mmm}$, $Z=4)$ и $D_{4 h}^{18}$ соответственно. Вторичные ПП $\eta_{i}$ определяются соотношениями (5). Фаза II $-\tau_{2} \neq 0, \eta_{1}<0$, $\tau_{1}=\eta_{2}=0$ (пр.гр. $\left.D_{4 h}^{18}\right)-$ появляется при $\beta_{1}>0$, фаза III $-\tau_{1} \neq 0, \eta_{1}>0, \eta_{2}=\tau_{2}=0$ - имеет место при $\beta_{1}<0$. Фаза III (пр. гр. $D_{4 h}^{17}$ ) характеризуется следующим набором функций $n_{i, L}: n_{1, \mathrm{I}}, n_{2, \mathrm{I}}=n_{3, \mathrm{I}}, n_{1, \mathrm{II}}, n_{2, \mathrm{II}}=n_{3, \mathrm{II}}$. Анализ зависимости $\beta_{1}$ от $b$ показывает, что на линии ФП второго рода $-\alpha_{1}=0\left(\alpha_{2} \geq 0\right)-$ коэффициент $\beta_{1}$ может 2 раза менять знак: при $B / T=0.422(b=1.422)$ 

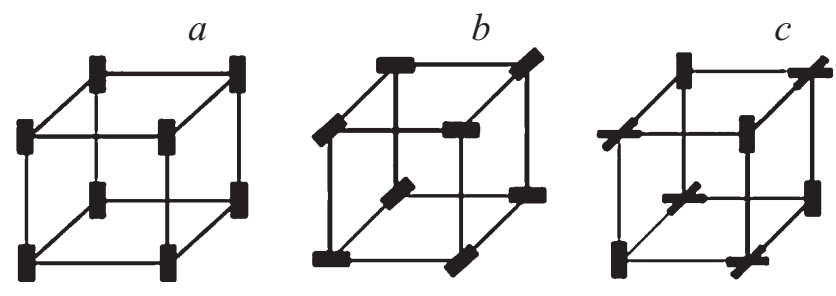

Pис. 2. Упорядочения, соответствующие фазам I (a), II (b), и III $(c)$.

и $B / T=1.121(b=2.121)$. Это означает, что рассматриваемая модель допускает существование двух мультикритических $N$-фазных точек $L$ и $M$ с координатами $B / T=0.422$ и $1.121(A / T=-1)$. Следствием этого является наличие очень близких по структуре и свойствам фаз (фаз II и $\mathrm{II}_{1}$ ) при существенно различных термодинамических условиях, что, в частности, свидетельствует о возможности так называемого „возврата в фазу“.

Вид диаграммы фазовых состояний в окрестности мультикритических $N$-фазных точек $L$ и $M$ можно определить, используя результаты, полученные в феноменологической теории ФП [29]. Условием того, что в окрестности точки $\alpha_{1}=0, \beta_{1}=0$ между фазами II $\left(0, \tau_{2}\right)$ и III $\left(\tau_{1}, 0\right)$ появится устойчивая фаза IV $\left(\tau_{1}, \tau_{2}\right)$, является $4 \alpha_{2} \beta_{2}-\delta_{12}^{2}>0$. В рассматриваемом случае это соотношение выполняется и в точке $L$, и в точке $M$. Поэтому точки $M$ и $L$ четырехфазные, в них соприкасаются четыре фазы: кубическая, две тетрагональные фаза III $\left(\tau_{1}, 0\right), D_{4 h}^{17}$ и фаза II (или $\left.\mathrm{II}_{1}\right)\left(0, \tau_{2}\right), D_{4 h}^{18}$, а также ромбическая фаза IV $\left(\tau_{1}, \tau_{2}\right), D_{2 h}^{23}$.

В области низких температур границы между фазами II-IV, IV-III и III-IV, IV-II 1 асимптотически сходятся к двум разным линиям: $B=-A$ и $B=-3 A$. Фаза IV граничит с фазами II, III и II $_{1}$ по линиям ФП второго рода. На диаграмме расположение границ фаз II-IV, IV-III и IV-III, IV-II 1 показано схематично. Согласно численным расчетам, область существования фазы IV очень узкая как между фазами II и III, так и между фазами III и $\mathrm{II}_{1}$.

Упорядочения, соответствующие фазовым состояниям I, II и III, показаны на рис. 2. Локальная симметрия узла во всех трех случаях различна. В первом (рис. 2, $a$ ) - это тетрагональная симметрия, во втором (рис. $2, b)$ - ромбическая, в третьем (рис. 2,c) - в обеих подрешетках тетрагональная, но во второй полученная посредством статистического усреднения. Структуры фаз $\mathrm{II}_{1}$ и II с симметрией $D_{4 h}^{18}$ аналогичны. Обе фазы возникают из симметричной при ФП второго рода. Основное отличие состоит в том, что при низких температурах (условно $T \rightarrow 0$ ) фаза II упорядочивается полностью: $n_{1, \mathrm{I}}=n_{1, \mathrm{II}} \rightarrow 0, n_{3, \mathrm{I}}=n_{2, \mathrm{II}} \rightarrow 0$, $n_{2, \mathrm{I}}=n_{3, \mathrm{II}} \rightarrow 1$ (рис. $\left.2, b\right)$, а в фазе $\mathrm{II}_{1} \quad\left(n_{1, \mathrm{I}} \rightarrow v>0\right.$, $n_{2, \mathrm{I}} \rightarrow 1-v<1, n_{3, \mathrm{I}} \rightarrow 0$, где $\left.v \approx(A+B) /(A+3 B)\right)$ в обеих подрешетках появляется небольшая $z$-компонента, которая сохраняется и при $T \rightarrow 0$.
В рассматриваемом случае область $T \sim 0-$ это область низких температур, в которой ПП достигает насыщения.

\section{4. Обсуждение}

Процесс формирования ФС можно представить следующим образом. В области $B<A(B<0)$ взаимодействия внутри подрешеток и между ними ориентируют частицы обеих подрешеток в одном направлении (фаза I, рис. $2, a)$. Поэтому в деформируемых решетках в случае если октаэдр (или частица) вытянуты, то фаза I также вытянута $(c / a>1)$, а если сжат, то и фаза I сжата $(c / a<1)$.

В области $A \lesssim B<0$ взаимодействие внутри подрешеток ориентирует частицы параллельно друг другу, a взаимодействие между подрешетками ориентирует их частицы ортогонально (рис. $2, b$ ). На линии $B=A$ $(A<0)$ энергии взаимодействия при параллельной и ортогональной ориентации частиц подрешеток оказываются одинаковыми (т.е. взаимодействие между подрешетками становится равным нулю). На этом сечении ориентация подрешеток меняется, и происходит ФП первого рода между фазами I и II. Несмотря на то что группа $D_{4 h}^{18}$ является подгруппой $D_{4 h}^{1}$, переход на линии $A=B, A<0$ может быть только переходом первого рода, так как фаза II возникает не из фазы I, а из другого (метастабильного) ФС, т.е. фазы I и II различаются своей „генеалогией“. В деформируемой решетке при переходе „растянутая“ $(c / a>1)$ фаза I трансформируется в „,жатую“ $(c / a<1)$ фазу II и, наоборот, „сжатая““ фаза I трансформируется в „растянутую“ фазу II.

В области $A<B \leq-A(A<0)$ упорядочения подрешеток ориентированы ортогонально друг другу (pис. 2,b). На линии $B=-A(A<0)$ фаза II через промежуточную фазу IV трансформируется в фазу III. Это превращение обусловлено изменением ориентации частиц внутри одной из подрешеток. В области $-A<B<-3 A \quad(A<0)$ частицы первой подрешетки остаются ориентированными одинаково, а частицы второй подрешетки ориентируются с равной вероятностью вдоль двух направлений, ортогональных ориентации первой подрешетки (рис. 2,c). В области $B>-3 A$ $(A<0)$ реализуется фаза $\mathrm{II}_{1}$. В этой фазе при низких температурах в плоскостях, параллельных оси $C_{4}$ (рис. $2, b$ ), имеется некоторый остаточный двумерный беспорядок в упорядочении орбиталей, что может несколько изменить магнитные и электрические свойства этой фазы по сравнению со свойствами фазы II.

Подобная модель в приближении взаимодействия ближайших соседей, т. е. на сечении $B=-A(A<0)$, анализировалась численными методами в работе [25]. Было показано, что на этом сечении имеет место ФП второго рода из кубической в тетрагональную фазу III. Учет взаимодействия с однородными деформациями позволил авторам несколько расширить исследуемую область 
$B<-A$. Полученная в [25] фазовая диаграмма, содержащая трикритическую и одну из мультикритических $N$-фазных точек, а также сечение $B=-A(A<0)$, совпадает с соответствующей частью диаграммы ФС (рис. 1).

Трехминимумная двухподрешеточная модель также может быть использована для исследования и описания ФП в ионно-молекулярных кристаллах. В кубической фазе кристаллов надперекисей $A \mathrm{O}_{2}(A=\mathrm{K}, \mathrm{Rb}, \mathrm{Cs})$, азидов $A_{\mathrm{I}} \mathrm{N}_{3} \quad\left(A_{\mathrm{I}}=\mathrm{Tl}, \mathrm{Rb}, \mathrm{Cs}\right)$ и дигидрофторида цезия $\mathrm{CsHF}_{2}$ анионы $\mathrm{O}_{2}^{-}$, а также линейные трехатомные анионы $\mathrm{N}_{3}^{-}, \mathrm{HF}_{2}^{-}$имеют три направления ориентации типа [001] [24,30,31]. При понижении температуры во всех этих соединениях наблюдаются ФП из кубической в тетрагональные фазы. В $\mathrm{CsO}_{2}$ и $\mathrm{KO}_{2}, \mathrm{RbO}_{2}$ это переходы $O_{h}^{1}(\mathrm{Pm} 3 \mathrm{~m})-D_{4 h}^{1}(P 4 / \mathrm{mmm})$ и $O_{h}^{5}(F m 3 m)-D_{4 h}^{17}(I 4 / m m m)$ соответственно. В обоих случаях реализуется упорядочение ферродисторсионного типа (ФС I) (рис. 2,a), характеризуемое вектором обратной решетки $\mathbf{k}=0$. Оно возникает при ФП первого рода из кубической фазы $O_{h}$. В азидах и дигидрофториде цезия имеет место переход из кубической $\operatorname{Pm} 3 m(Z=1)$ в антиферродисторсионно упорядоченную фазу симметрии $I 4 / m c m(Z=4)$ (рис. 2,b). В кристаллах $\mathrm{CsHF}_{2}$ и $\mathrm{CsN}_{3}$ это ФП первого рода.

Описание орбитального упорядочения как ФП типа порядок-беспорядок опирается на предположение о том, что в симметричной фазе формируется ансамбль искаженных анионных октаэдров. Оценим, насколько такие представления могут быть общими для всей совокупности перовскитов, содержащих ЯТ-катионы. Запишем энергию одиночного тетрагонально искаженного вследствие снятия электронного вырождения октаэдра в кубической решетке перовскита

$$
U=\delta\left(x_{0}-z_{0}\right)+4 k_{B}\left(\frac{a}{2}-x_{0}\right)^{2}+2 k_{B}\left(\frac{a}{2}-z_{0}\right)^{2} .
$$

Первый член представляет понижение электронной энергии при расщеплении $e_{g}$-уровня катиона $B$, второй и третий описывают упругую энергию искаженного октаэдра. В этом выражении $z_{0}$ и $x_{0}$-- расстояния между центральным катионом $B$ и анионом соответственно вдоль тетрагональной оси и в плоскости, ортогональной этой оси, $a$ - параметр неискаженной кубической ячейки, $k_{B}-$ „константа упругости“ катион-анионной связи. Минимизируя $U$ по $z_{0}$ и $x_{0}$, получаем

$$
x_{0}=\frac{a}{2}-\frac{\delta}{8 k_{B}}, \quad z_{0}=\frac{a}{2}+\frac{\delta}{4 k_{B}} .
$$

Мы имеем возможность оценить константу $\delta$ по соотношению $z_{0}-x_{0}=3 \delta / 8 k_{B}$, воспользовавшись экспериментальными данными для $z_{0}$ и $x_{0}$ : в $\mathrm{LaMnO}_{3}$ $z_{0}=2.13 \AA, x_{0}=1.92 \AA \quad[17]$, в $\mathrm{KCrF}_{3} \quad z_{0}=2.28 \AA$, $x_{0}=1.98 \AA[18]$. Для $k_{B}$ в работах [32,33], посвященных вычислению параметров решетки, было принято $k_{B} \approx \gamma n_{B}$, где $n_{B}-$ валентность катиона $B$. Используя данные по объемной сжимаемости оксидов семейства перовскитов, собранные в [34], получаем усредненное значение $\gamma \approx 70 \mathrm{~N} / \mathrm{m}$ и значения $\delta \approx 1.2 \cdot 10^{-8} \mathrm{~N}$ для $\mathrm{LaMnO}_{3}$ и $\delta \approx 1.1 \cdot 10^{-8} \mathrm{~N}$ для $\mathrm{KCrF}_{3}$. Это позволяет определить с помощью (7) изменение энергии $U_{\mathrm{I}}$, отнесенное к одной частице октаэдра, обусловленное деформацией октаэдра. Для $\mathrm{LaMnO}_{3} U_{\mathrm{I}} \approx-0.2 \cdot 10^{-19} \mathrm{~J}$, для $\mathrm{KCrF}_{3} U_{1} \approx-0.23 \cdot 10^{-19} \mathrm{~J}$. Отсюда следует, что при температурах ниже $1.45 \cdot 10^{3} \mathrm{~K}\left(\mathrm{LaMnO}_{3}\right)$ и $1.67 \cdot 10^{3} \mathrm{~K}$ $\left(\mathrm{KCrF}_{3}\right)$ можно ожидать стабильного существования деформированных октаэдров и в других перовскитах с ЯТ-катионами и применимости рассмотренной статистической модели для описания орбитального упорядочения.

Для описания всей совокупности антиферродисторсионных ФС, возможных по симметрии, необходимы лишь две силовые константы: $A$ и $B(2)$. Эти константы характеризуют взаимодействия как внутри подрешеток, так и между ними. Приближению, в котором учитывается взаимодействие ближайших соседей, соответствует термодинамический путь вдоль луча $A=-B(A<0)$. В этом случае будет иметь место ФП второго рода из кубической в тетрагональную фазу III. Однако в рассматриваемых кристаллах наблюдается ФП первого рода между фазами $C$ и II. Из рис. 1 следует, что для этого необходимо выполнение соотношения $A<B<0.5 A, A<0$.

Учет взаимодействия ПП с однородными одноосными деформациями приводит к перенормировке в сторону уменьшения константы $B$, что дало возможность в рамках приближения взаимодействия ближайших coceдей описать и ФП из кубической в тетрагональную фазу II [25]. Специфика рассматриваемых соединений в том, что октаэдры заметно деформированы даже в кубической фазе. Поэтому важную роль при формировании упорядоченных фазовых состояний играют значительные по величине локальные деформации. Именно они во многом определяют и величину однородных деформаций.

Учет локальных деформаций приводит к перенормировке константы $A$ и косвенным образом (через однородные деформации) к перенормировке константы $B$. Согласно приближенным оценкам, величины перенормировки (в сторону уменьшения) констант $B$ и $A$ пропорциональны друг другу, но вторая приблизительно на $20 \%$ больше первой. Это означает, что для систем, в которых основным является упругое взаимодействие деформированных октаэдров с ближайшими соседями, будет иметь место соотношение $A \lesssim B<0,|B|>0.5|A|$. Поэтому, согласно данным рис. 1 , при понижении температуры будет происходить ФП первого рода из фазы $C$ в фазу II. Для таких систем наиболее предпочтительным является упорядочение антиферродисторсионного (антиферроорбитального) типа, возникающее при ФП первого рода. Это также объясняет отсутствие во всех упомянутых соединениях фаз III и IV. То же самое имеет место и в обсуждаемых ионно-молекулярных кристаллах, поскольку в них локальные деформации 
также велики и имеет место сильное взаимодействие с ближайшими соседями.

Таким образом, по-видимому, одной из основных причин того, что в рассматриваемых соединениях часто наблюдается именно антиферродисторсионное упорядочение типа ФС II [5,24,35], является наличие локально деформированных октаэдров, а также сильного упругого (через деформации связей) взаимодействия с ближайшими соседями в подсистеме ЯТ-катионов.

\section{5. Заключение}

В рассматриваемых соединениях тетрагонально искаженные вследствие эффекта Яна-Теллера анионные октаэдры могут появиться в симметричной кубической фазе при температурах, значительно превышающих температуры возникновения дальнего порядка. Поэтому появление упорядочений в подсистеме орбитально деформированных октаэдров действительно можно трактовать как переходы типа порядок-беспорядок, обусловленные их ориентационным упорядочением вдоль трех направлений. Анализ и описание таких ФП, характеризуемых $\mathbf{k}=0$ и $\mathbf{k}=(1 / 2,1 / 2,1 / 2)$, выполнены на основе трехминимумной двухподрешеточной модели.

Диаграмма фазовых состояний этой модели построена на всей фазовой плоскости, т.е. известны фазовые состояния при любых соотношениях между параметрами модели и термодинамическими параметрами. Это дает возможность получить представление о ФС и статистико-термодинамических свойствах соединений, которые существенно отличаются друг от друга, а также проследить, как меняются характеристики системы при движении вдоль различных термодинамических путей. Учет локальных деформаций и деформируемости решетки (в первом приближении) приводит к перенормировке модельных констант $A$ и $B$, при этом общий вид диаграммы не меняется.

Полученная диаграмма является базовой для любых соединений, которые могут описываться трехминимумной двухподрешеточной моделью. В таких соединениях могут возникнуть четыре типа упорядоченных фаз, одна из которых является собственно сегнетоэластической (упорядочение ферродисторсионного типа, характеризуемое $\mathbf{k}=0$ ), а остальные три - фазы антиферродисторсионного типа, характеризуемые $\mathbf{k}=(1 / 2,1 / 2,1 / 2)$. Из сопоставления полученных результатов с данными структурных исследований ФП, обусловленных орбитальными упорядочениями в перовскитах и линейных анионов в ионно-молекулярных кристаллах, следует, что на основе этой модели можно описать весь набор возникающих упорядочений.

\section{Список литературы}

[1] Y. Tokura, N. Nagaosa. Science 288, 462 (2000).

[2] Ю.А. Изюмов, Я.Н. Скрябин. УФН 171, 121 (2001).
[3] J.B. Goodenough. Annu. Rev. Mater. Sci. 28, 1 (1998).

[4] M. Kataoka, J. Kanamory. J. Phys. Soc. Jpn. 32, 113 (1972).

[5] К.И. Кугель, Д.И. Хомский. УФН 138, 621 (1982).

[6] Д. Гуденаф. Магнетизм и химическая связь. Металлургия, M. (1968). 325 c.

[7] J.-S. Zhou, J.B. Goodenough. Phys. Rev. B 60, R 15002 (1999).

[8] J.D. Dunitz, L.E. Orgel. J. Phys. Chem. Solids 3, 20 (1957).

[9] S. Margadonna, G. Karotsis. J. Amer. Chem. Soc. 128, 16436 (2006).

[10] V. Kaiser, M. Otto, F. Binder, D. Babel. Z. Anorg. Allg. Chem. 585, 93 (1990).

[11] M. Hutchings, E.J. Samuelsen, G. Shirane, K. Hirakawa. Phys. Rev. 188, 919 (1969).

[12] M.A. Hepworth, K.H. Jack. Acta. Cryst. 10, 345 (1957).

[13] P. Daniel, A. Bulou, M. Rousseau, J. Nouet. Phase Trans. 33, 91 (1991).

[14] J. Rodriguez-Carvajal, M. Hennion, F. Moussa, A.H. Moudden, L. Pinsard, A. Revcolevschi. Phys. Rev. B 57, 5259 (1998).

[15] A.A. Belik, S. Iikubo, T. Yokosawa, K. Kodama, N. Igawa, S. Shamoto, M. Azuma, M. Takano, K. Kimoto, Y. Matsui, E. Takayama-Muromachi. J. Am. Chem. Soc. 129, 971 (2007).

[16] A. Sartbaeva, S.A. Wells, M.E. Thorpe, E.S. Božin, S.J.L. Billinge. Phys. Rev. Lett. 99, 155503 (2007).

[17] J. Garsia, G. Subfas, M.C. Sánchez, J. Blasco. Phys. Scripta T 115, 594 (2005).

[18] S. Margadonna, G. Karotsis. J. Mater. Chem. 17, 2013 (2007).

[19] B. Halperin, R. Englman. Phys. Rev. B 3, 1698 (1971).

[20] H.T. Stokes, D.M. Hatch. Isotropy subgroup of 230 crystallografic space groups. World Scientific, Singapore (1988). $573 \mathrm{p}$.

[21] М.П. Ивлиев. ФТТ 51, 1472 (2009).

[22] P.J. Wojtowicz. Phys. Rev. 116, 32 (1959).

[23] М.П. Ивлиев, В.П. Сахненко ФТТ 28, 632 (1986).

[24] Н. Парсонидж, Л. Стейвли. Беспорядок в кристаллах. Мир, М. (1982). Т. 1. 434 с.

[25] K.H. Hock, G. Schroder, H. Thomas. Z. Phys. B 30, 403 (1978).

[26] W. Gorsky. Z. Phys. 50, 64 (1928).

[27] W.L. Bragg, E.J. Williams. Proc. Roy. Soc. A 145, 699 (1934).

[28] М.П. Ивлиев, В.П. Сахненко. Фазовые переходы типа порядок-беспорядок, описываемые двумя многокомпонентными параметрами порядка (приближение среднего поля). Деп. в ВИНИТИ № 3418-89 от 19.05.89, Ростов. гос. ун-т, Ростов н/Д (1989). 35 с.

[29] В.П. Сахненко, В.М. Таланов. ФТТ 21, 2435 (1979).

[30] M. Rosenfeld, M. Ziegler, W. Konzig. Helv. Phys. Acta 5, 298 (1978).

[31] A. Fuith. Phase Trans. 62, 1 (1997).

[32] В.П. Сахненко, Е.Г. Фесенко, А.Т. Шуваев, Е.Т. Шуваева, Г.А. Гегузина. Кристаллография 17, 316 (1972).

[33] В.П. Сахненко, Н.В. Дергунова, Л.А. Резниченко. Энергетическая кристаллохимия твердых растворов соединений кислородно-октаэдрического типа и моделирование пьезокерамических материалов. Изд-во Ростов. гос. пед. ун-та, Ростов н/Д (1999). 322 с.

[34] M.W. Lufaso, S.J. Mugavero III, V.R. Gemmill, Y. Lee, T. Vogt, H.-C. zur Loge. J. Alloys Comp. 433, 91 (2007).

[35] M.W. Lufaso, P.M. Woodward. Acta Cryst. B 60, 10 (2004). 